\title{
Investigating the cohomological dimensions of $\mathcal{M}_{g}$
}

\author{
Gabriele Mondello \\ Dipartimento di Matematica \\ "Sapienza" Università di Roma \\ Piazzale Aldo Moro 5, 00185 Roma, Italy \\ mondello@mat.uniroma1.it
}

Received 3 March 2015

Accepted 18 April 2016

Published 7 June 2016

\begin{abstract}
We discuss the problem of determining the de Rham, Dolbeault and algebraic cohomological dimension of $\mathcal{M}_{g}$, focusing on possible strategies of attack and then concentrating on exhaustion functions. In the final section, we explain how these techniques can be employed to provide a nontrivial upper bound for the Dolbeault cohomological dimension of $\mathcal{M}_{g}$.
\end{abstract}

Keywords: Riemann surfaces; moduli space; cohomological dimension; translation surfaces.

Mathematics Subject Classification 2010: 32G15, 32F10, 30F30

\section{Moduli Space of Riemann Surfaces}

Let $g \geq 2$ and denote by $\mathcal{M}_{g}$, the moduli space of compact connected Riemann surfaces of genus $g$ up to isomorphism.

The space $\mathcal{M}_{g}$ can be viewed as an orbifold (see for instance [1]). Indeed, if $x=[S] \in \mathcal{M}_{g}$ represents the isomorphism class of the Riemann surface $S$, points in a neighborhood of $x$ encode deformations of complex structures on $S$ up to isomorphism. It is well-known that, such deformations are parametrized by an open subset $U$ of $\mathbb{C}^{3 g-3}$ (see [6] or, for instance, the book [5]) and that the finite automorphism group of $S$ acts on $U$. As a consequence, a neighborhood of $x$ in $\mathcal{M}_{g}$ looks like $U / \operatorname{Aut}(S)$ and so $\mathcal{M}_{g}$ can be given the structure of a complex-analytic orbifold. From the algebraic point of view, $\mathcal{M}_{g}$ can be endowed with the structure of smooth Deligne-Mumford stack [11]. In fact, it is well-known [19], that $\mathcal{M}_{g}$ is a global quotient of a smooth complex quasi-projective variety $\widetilde{\mathcal{M}}_{g}$ by the action of a finite group $G$. Hence, sheaves and sections of sheaves on $\mathcal{M}_{g}$ can be defined as $G$-equivariant sheaves and sections on $\widetilde{\mathcal{M}}_{g}$.

Analogous considerations hold for the moduli space $\mathcal{M}_{g, k}$ of compact connected Riemann surfaces $S$ with $k$ distinct marked points $p_{1}, \ldots, p_{k} \in S$, provided the 
hyperbolicity condition $2 g-2+k>0$ is satisfied and so all automorphism groups $\operatorname{Aut}\left(S, p_{1}, \ldots, p_{k}\right)$ are finite.

\subsection{On the cohomology of the moduli space of Riemann surfaces}

Many papers are dedicated to the study of the singular cohomology of $\mathcal{M}_{g}$.

In low degrees, genus-independent computations are due to Powell [45] and Harer [20] for $H_{1}$, to Harer [20, 23] for $H_{2}$ and $H_{3}$, to Edidin [14] and Polito [44] for $\mathrm{H}_{4}$ and $g \geq 8$.

As for low-genera computations, de Rham cohomology is trivial for $g=2$. A complete calculation in genus 3 is due to Looijenga [32] and in genus 4 to Tommasi [49], but the full determination of $H_{d R}^{*}\left(\mathcal{M}_{g}\right)$ seems out of reach for $g$ large. Further computations are available for the moduli spaces $\mathcal{M}_{g, k}$ of surfaces with marked points.

Still due to Harer are the following two major results, which here, we state in a simplified version.

Theorem 1.1 ([22]). Let $2 g-2+k>0$. Then $H^{d}\left(\widetilde{\mathcal{M}}_{g, k} ; \mathbb{L}\right)=0$ for every local system $\mathbb{L}$ and every $d>d(g, k)$, where

$$
d(g, k)= \begin{cases}n-3 & \text { if } g=0 \text { and } n \geq 3 \\ 4 g-5 & \text { if } g \geq 2 \text { and } n=0 \\ 4 g-4+n & \text { if } g \geq 1 \text { and } n \geq 1 .\end{cases}
$$

Hence, $H_{d R}^{d}\left(\mathcal{M}_{g, k} ; \mathbb{V}\right)=0$ for every $\mathbb{C}$-local system $\mathbb{V}$ and every $d>d(g, k)$.

Actually, Harer's result is much more powerful. In particular, it implies that there exists a $G$-invariant local system $\mathbb{S}$ on $\widetilde{\mathcal{M}}_{g, k}$ such that $H_{d R}^{d(g, k)}\left(\mathcal{M}_{g, k} ; \mathbb{S}\right) \neq 0$ and so that the bound is sharp.

Theorem 1.2 (Stability [21]). Fix a degree $d>0$. Then there exists an integer $g(d) \approx 3 d$, such that $H^{d}\left(\mathcal{M}_{g+1} ; \mathbb{Z}\right) \cong H^{d}\left(\mathcal{M}_{g} ; \mathbb{Z}\right)$ for all $g \geq g(d)$.

The stability threshold was improved by Ivanov [27] to $g(d) \approx 2 d$ and then by Boldsen [10] to the approximately optimal $g(d) \approx \frac{3}{2} d$. Moreover, the full rational stable cohomology was determined by Madsen-Weiss [35].

Though very important, de Rham cohomology and even Hodge theory do not always capture subtleties of the holomorphic geometry of a complex manifold, which are encoded by Dolbeault cohomology: for instance, deformations of complex structures, deformations of analytic coherent sheaves, deformation of holomorphic maps or of holomorphic subvarieties and so on. On the other hand, since de Rham cohomology is isomorphic to singular cohomology with rational coefficients, its computation can benefit of a whole array of topological tools, which are unavailable, if one wishes to work with Dolbeault cohomology.

We emphasize that most genus-independent results in de Rham cohomology of $\mathcal{M}_{g}$ are obtained via purely transcendental methods. So is, for instance, for the 
determination of the orbifold Euler characteristic by Harer-Zagier [24], Penner [41] and Kontsevich [30]. Even the most algebro-geometric approaches (for example [4]), seem to rely on the topological computations of $H^{1}$ and $H^{2}$ and on Theorem 1.1. Thus, it is not surprising that Dolbeault cohomology of $\mathcal{M}_{g}$ remains almost completely unknown. In particular, no analogue of Theorem 1.2 exists at present in the Dolbeault setting, whereas Theorem 1.1 is replaced by the following.

Problem 1.3 (Looijenga). Show that, for every holomorphic vector bundle $E$ on $\mathcal{M}_{g}$, we have $H_{\bar{\partial}}^{0, q}\left(\mathcal{M}_{g} ; E\right)=0$ for $q>g-2$.

Evidence supporting Looijenga's conjecture is basically of four types:

(1) it is confirmed for $g \leq 5$ (by classical arguments for $g=2,3$ and by FontanariPascolutti [15] for $g=4,5)$;

(2) it would imply Diaz's result [13]: compact holomorphic subvarieties of $\mathcal{M}_{g}$ have dimension at most $g-2$;

(3) it would imply Looijenga's vanishing [33] of tautological classes on $\mathcal{M}_{g}$ beyond degree $g-2$;

(4) it would imply Harer's Theorem 1.1 through the Dolbeault-to-de Rham spectral sequence $H_{\bar{\partial}}^{0, q}\left(\mathcal{M}_{g} ; \Omega^{p, 0} \otimes \mathbb{V}\right) \Rightarrow H_{d R}^{p+q}\left(\mathcal{M}_{g} ; \mathbb{V}\right)$.

For the (partially) compactified moduli space of Riemann surfaces, the analogous of Looijenga's vanishing is proven by Ionel [26] and Graber-Vakil [18], whereas an analogous bound as in Theorem 1.1 is derived in [37]. A more detailed discussion about the above results, the employed techniques, Looijenga's problem and stratification techniques can be found in [39].

Considerations analogous to those expressed about Dolbeault cohomology hold for the cohomology of algebraic coherent sheaves on $\mathcal{M}_{g}$.

\section{Investigating the Cohomological Dimension}

\subsection{Basic properties}

Let $\mathfrak{F}$ be one of the following three categories:

$(\mathrm{dR}) \mathfrak{F}=\mathrm{dR}$ the category of $\mathbb{C}$-local systems of coefficients (i.e. flat complex vector bundles) over the category $\mathfrak{C}$ of smooth manifolds with smooth maps;

(Dol) $\mathfrak{F}=$ Dol the category of holomorphic vector bundles, ${ }^{\text {a }}$ over the category $\mathfrak{C}$ of complex manifolds with holomorphic maps;

(alg) $\mathfrak{F}=$ alg the category of algebraic vector bundles, ${ }^{\text {a }}$ over the category $\mathfrak{C}$ of complex algebraic varieties with algebraic maps.

For every object $X$ of $\mathfrak{C}$, denote by $\mathfrak{F}_{X}$ the subcategory of $\mathfrak{F}$ of sheaves on $X$.

\footnotetext{
${ }^{a}$ Actually, more appropriate and functorial definitions require the category of analytic coherent sheaves (Dol) and of algebraic coherent sheaves (alg).
} 
Definition 2.1. The $\mathfrak{F}$-cohomological dimension of $X$ is the greatest integer $d \geq 0$ for which there exists a sheaf $\mathcal{F} \in \mathfrak{F}_{X}$, such that $H^{d}(X ; \mathcal{F}) \neq 0$. We denote it by $\operatorname{coh}_{-\operatorname{dim}_{\mathfrak{F}}}(X)$.

Since, we will mostly focus on de Rham (dR) and Dolbeault (Dol) cohomological dimensions, we leave the algebraic (alg) cohomological dimension aside for a moment. In the following table, a few immediate properties of these invariants are displayed.

\begin{tabular}{|c|c|}
\hline $\begin{array}{l}\text { DE RHAM COHOMOLOGY } \\
X \text { connected manifold }\end{array}$ & $\begin{array}{c}\text { Dolbeault cohomology } \\
X \text { connected complex manifold }\end{array}$ \\
\hline $\begin{array}{c}X \text { compact } \Longleftrightarrow \operatorname{dim}_{\mathbb{R}}(X)=\text { coh- } \operatorname{dim}_{d R}(X) \\
X \text { contractible } \Longrightarrow X \text { acyclic }\end{array}$ & $\begin{array}{c}X \text { compact } \Longleftrightarrow \operatorname{dim}_{\mathbb{C}}(X)=\text { coh-dim } \operatorname{dol}_{D o l}(X) \\
X \text { Stein } \Longrightarrow X \text { acyclic }\end{array}$ \\
\hline $\begin{array}{c}F \rightarrow Y \rightarrow X \text { proper smooth submersion } \\
\text { coh-dim }_{d R}(Y)=\text { coh-dim } d R(X)+\text { coh-dim }_{d R}(F)\end{array}$ & $\begin{array}{c}F \rightarrow Y \rightarrow X \text { proper holomorphic submersion } \\
\text { coh-dim }_{D o l}(Y)=\text { coh-dim } \\
\text { Dol }\end{array}$ \\
\hline $\begin{array}{c}Y \rightarrow X \text { unramified finite cover } \\
\Longrightarrow \text { coh- } \operatorname{dim}_{d R}(Y)=\text { coh- } \operatorname{dim}_{d R}(X)\end{array}$ & $\begin{array}{c}Y \rightarrow X \text { (unramified) finite cover } \\
\Longrightarrow \text { coh-dim } \operatorname{dol}_{D o l}(Y)=\text { coh-dim } \operatorname{diol}_{D o l}(X)\end{array}$ \\
\hline
\end{tabular}

As an example, we prove one such property which will have a special meaning.

Lemma 2.1. Let $\mathfrak{C}$ be one of the three above categories and let $\pi: Y \rightarrow X$ be a morphism in $\mathfrak{C}$, which is a finite surjective unramified cover. Then:

(a) for every $\mathcal{G} \in \mathfrak{F}_{Y}$ the push-foward $\pi_{*} \mathcal{G}$ belongs to $\mathfrak{F}_{X}$;

(b) for every $\mathcal{F} \in \mathfrak{F}_{X}$ the pull-back $\pi^{*} \mathcal{F}$ belongs to $\mathfrak{F}_{Y}$;

(c) $\operatorname{coh}-\operatorname{dim}_{\mathfrak{F}}(Y)=\operatorname{coh}-\operatorname{dim}_{\mathfrak{F}}(X)$.

Proof Assertions (a) and (b) are clear, so we focus on property (c). Since $\pi$ is finite unramified and surjective, $\mathcal{O}_{X}$ is a direct summand of $\pi_{*} \mathcal{O}_{Y}$. Thus, $H^{q}(X ; \mathcal{F})$ is a direct summand of $H^{q}\left(X, \mathcal{F} \otimes_{\mathcal{O}_{X}} \pi_{*} \mathcal{O}_{Y}\right)=H^{q}\left(Y, \pi^{*} \mathcal{F}\right)$ and so $\operatorname{coh}_{-\operatorname{dim}_{\mathfrak{F}}}(X) \leq \operatorname{coh}_{-\operatorname{dim}_{\mathfrak{F}}}(Y)$. On the other hand, $H^{q}(Y, \mathcal{G})=H^{q}\left(X, \pi_{*} \mathcal{G}\right)$ implies that $\operatorname{coh}-\operatorname{dim}_{\mathfrak{F}}(Y) \leq \operatorname{coh}-\operatorname{dim}_{\mathfrak{F}}(X)$.

We remark that de Rham (respectively Dolbeault, or algebraic) cohomology can be defined also for the category $\mathfrak{F}$ of complex local systems on orbifolds (respectively of holomorphic vector bundles on complex-analytic orbifolds, or of algebraically coherent locally free sheaves on Deligne-Mumford stacks over $\mathbb{C}$ ).

Remark 2.1. The above lemma shows that, if $X=[Y / G]$ is a global quotient of $Y$ by the action of a finite group, then $\operatorname{coh}-\operatorname{dim}_{\mathfrak{F}}(X)=\operatorname{coh}-\operatorname{dim}_{\mathfrak{F}}(Y)$.

Notice that, if $M=B \Gamma$ for a virtually torsion-free discrete group $\Gamma$, then coh- $\operatorname{dim}_{\mathrm{dR}}(M)$ coincides with the virtual cohomological dimension of $\Gamma$ (see [7]). In this language, Theorem 1.1 can be rephrased as follows.

Theorem 2.1 (Harer [22]). Coh-dim $\operatorname{dR}_{\mathrm{dR}}\left(\mathcal{M}_{g, k}\right)=d(g, k)$. 
Also Problem 1.3 has the following rephrasing.

Problem 2.2 (Looijenga). Show that coh-dim $\operatorname{Dol}_{\text {Dol }}\left(\mathcal{M}_{g}\right) \leq g-2$.

We remark that the Dolbeault-to-de Rham spectral sequence together with Harer's result coh- $\operatorname{dim}_{\mathrm{dR}}\left(\mathcal{M}_{g}\right)=4 g-5$ already implies that coh-dim $\operatorname{Dol}\left(\mathcal{M}_{g}\right) \geq$ $g-2$. Thus, Looijenga's problem is equivalent to exactly determining the Dolbeault cohomological dimension of $\mathcal{M}_{g}$.

\subsection{Strategies of attack}

Suppose that, we want to bound the de Rham cohomological dimension of a noncompact manifold $M$. We review four basic ideas to attack this problem.

$\left(1_{\mathrm{dR}}\right)$ Let $\mathfrak{U}=\left\{U_{i}\right\}_{i \in \mathfrak{I}}$ of $M$ be a locally finite open cover, such that

— for every nonempty finite subset $I=\left\{i_{0}, \ldots, i_{p}\right\} \subseteq \mathfrak{I}$ of cardinality $p+1$, the de Rham cohomological dimension of $U_{I}:=U_{i_{0}} \cap \cdots \cap U_{i_{p}}$ is at most $d-p$ (and so in particular $U_{I}=\emptyset$ for $|I|>d+1$ ).

Then the spectral sequence $\bigoplus_{|I|=p} H_{\mathrm{dR}}^{q}\left(U_{I} ; \mathbb{V}\right) \Rightarrow H_{\mathrm{dR}}^{p+q}(M ; \mathbb{V})$ for every

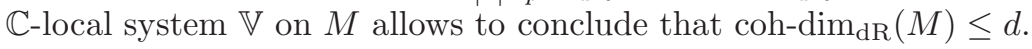

$\left(2_{\mathrm{dR}}\right)$ Let $M^{0} \cup M_{1} \cup \cdots \cup M^{r}$ be a stratification of $M$, such that

(a) the closure of $M^{q}$ is $\bigcup_{i \geq 0} M^{q+i}$ and each $M^{q+i}$ has a neighborhood in $\overline{M^{q}}$ that retracts by deformation onto $M^{q+i}$;

(b) $M^{q}$ is a locally closed smooth submanifold of $M$ of codimension $q$;

(c) the de Rham cohomological dimension of $M^{q}$ is at most $d-q$.

Then (a) allows to thicken each $M^{q}$ to produce an open cover $\mathfrak{U}=$ $\left\{U_{0}, \ldots, U_{r}\right\}$ of $M$ in such a way that

(i) $U_{q}$ contains $M^{q}$ and it deformation retracts onto $M^{q}$;

(ii) for every $q$ and $0=i_{0}<i_{1}<\cdots<i_{p}$, the open subset $U_{q} \cap U_{q+i_{1}} \cap \cdots \cap$ $U_{q+i_{p}}$ is homotopy equivalent to a fibration over $M^{q+i_{p}}$ with compact fibers of dimension $\left(i_{1}-1\right)+\left(i_{2}-i_{1}-1\right)+\cdots+\left(i_{p}-i_{p-1}-1\right)=$ $i_{p}-p$, and so they have de Rham cohomological dimension at most $\left[d-\left(q+i_{p}\right)\right]+\left(i_{p}-p\right) \leq d-p$.

By $\left(1_{\mathrm{dR}}\right)$, it follows that coh- $\operatorname{dim}_{\mathrm{dR}}(M) \leq d$.

$\left(3_{\mathrm{dR}}\right)$ Assume that $M$ retracts by deformation onto a CW complex $K$ of dimension d. Using the isomorphism between de Rham and singular homology, one can conclude that the de Rham cohomological dimension of $M$ is at most $d$.

$\left(4_{\mathrm{dR}}\right)$ Suppose that one can exhibit a $C^{2}$ proper function $\xi: M \rightarrow \mathbb{R}$ bounded below (also known as exhaustion funtion) with isolated critical points and such that the Hessian at its critical points is nondegenerate and of index at most $d$. Then Morse theory tells us that $M$ is homotopy equivalent to a CW complex of dimension at most $d$ and so coh-dim $\operatorname{dR}_{\mathrm{dR}}(M) \leq d$. 
Suppose, now that, we have a complex manifold $X$ and we would like to bound its Dolbeault cohomological dimension. While strategy (1) can be translated word by word in this setting, variant (2) needs much more care. We do not have a handy analogue of (3) but approach (4) can be adapted as follows.

$\left(4_{\text {Dol }}\right)$ Exhibit an exhaustion function $\xi: X \rightarrow \mathbb{R}$, such that at every point $x \in X$ the complex Hessian $i \partial \bar{\partial} \xi$ restricts to a positive-definite Hermitian form on a subspace $W_{x} \subset T_{x} X$ of codimension $d$ (at least in a suitable distributional sense). By Andreotti-Grauert [3], it follows that coh-dim $\operatorname{Dol}(X) \leq d$.

We stress that, despite the formal analogies between $\left(4_{\mathrm{dR}}\right)$ and $\left(4_{\mathrm{Dol}}\right)$, the proof by Andreotti-Grauert is not Morse-theoretic: it passes through producing a complete Kähler metric on $X$ with respect to which all but $d$ eigenvalues of $i \partial \bar{\partial} \xi$ are one and the remaining $d$ eigenvalues of $i \partial \bar{\partial} \xi$ are greater than $-\varepsilon$, and then applying Bochner technique [8, 9] as in Kodaira-Akizuki-Nakano's proof [29, 2]. See also the book [12].

Similar considerations on strategies (1) and (2) hold for a complex algebraic variety $Y$, and its algebraic cohomological dimension, provided the cover $\mathfrak{U}$ is open in the Zariski topology. More specifically, a popular way to pursue these strategies in the algebraic setting is the following (see also [46]).

$\left(1_{\mathrm{alg}}\right)$ Let $\bar{Y}$ be a smooth compactification of $Y$ in which $\partial Y:=\bar{Y} \backslash Y$ is a divisor. Suppose that $D_{0}, \ldots, D_{d} \subset \bar{Y}$ are effective ample divisors, such that $\bigcap_{i=0}^{d} D_{i} \subseteq \partial Y$. Then $Y$ is union of $d+1$ affine subsets $Y \backslash D_{0}, \ldots, Y \backslash D_{d}$ and so coh-dimalg $(Y) \leq d$.

$\left(2_{\mathrm{alg}}\right)$ Let $Y^{0} \supset \cdots \supset Y^{d}$ be a stratification of $Y$, such that $\overline{Y^{q}}=\bigcup_{i \geq 0} Y^{q+i}$ and $Y_{q}$ has codimension $q$ in $Y$. Suppose that each $Y^{q}$ is affine. Then coh-dimalg $(Y) \leq d$.

Since affines are Stein, $\left(1_{\mathrm{alg}}\right)$ implies $\left(1_{\mathrm{Dol}}\right)$.

Finally, strategy (4) has the following incarnation in the algebraic setting.

$\left(4_{\text {alg }}\right)$ Exhibit a smooth compactification $\bar{Y}$ of $Y$ and a holomorphic line bundle $L \rightarrow \bar{Y}$ with a Hermitian metric $h$ such that

(i) the added points at infinity $\partial Y:=\bar{Y} \backslash Y$ form a Cartier divisor;

(ii) there exists a holomorphic section $\sigma$ of $L$ that vanishes exactly on $\partial Y$;

(iii) at every point $y \in \bar{Y}$ the complex Hessian $-i \partial \bar{\partial} \log \|s\|_{h}^{2}$ restricts to a positive-definite Hermitian form on a subspace $W_{y} \subset T_{y} \bar{Y}$ of codimension $d$, where $s$ is any nonvanishing holomorphic section of $L$ defined on a neighborhood of $y$.

A suitable modification of the argument by Andreotti-Grauert [3] similar to Girbau's $[16,17]$ (see Demailly [12]) implies that coh-dimalg $(Y) \leq d$.

By letting $\xi:=-\log \|\sigma\|_{h}^{2}$, we easily see that $\left(4_{\mathrm{alg}}\right)$ implies $\left(4_{\mathrm{Dol}}\right)$. 


\section{Dolbeault Cohomological Dimension of $\mathcal{M}_{g}$ via Exhaustion Functions}

\subsection{Hyperbolic surfaces}

Suppose, we want to bound the Dolbeault cohomological dimension of the moduli space $\mathcal{M}_{g}$ of compact connected Riemann surfaces of genus $g \geq 2$ by taking approach $\left(4_{\mathrm{Dol}}\right)$ described in Sec. 2.2. We have then to look for natural, and possibly geometric, exhaustion functions on $\mathcal{M}_{g}$.

Remark 3.1. Using the uniformization theorem [43], one can endow every compact connected Riemann surface $S$ of genus $g \geq 2$ with its Poincaré hyperbolic metric. Moreover, such a hyperbolic metric depends real-analytically on the complex structure on $S$.

Definition 3.1. For every nontrivial simple closed curve $\gamma$ on $S$, we define $\ell_{\gamma}^{\text {hyp }}(S)$ as the hyperbolic length of the unique geodesic on $S$ freely homotopic to $\gamma$ and the systole $\ell_{\mathrm{sys}}^{\mathrm{hyp}}(S)$ as the hyperbolic length of the shortest nontrivial closed curve on $S$.

Almost by definition, the variation of the hyperbolic length functions (which are well-defined locally on $\mathcal{M}_{g}$ ) real-analytically depends on the variation of complex structure on the Riemann surface. Moreover, the following is rather easy to check.

Lemma 3.1. The function $\ell_{\mathrm{sys}}^{\mathrm{hyp}}: \mathcal{M}_{g} \rightarrow \mathbb{R}$ is locally the minimum of finitely many hyperbolic length functions. Hence, $\ell_{\mathrm{sys}}^{\mathrm{hyp}}$ is locally Lipschitz.

The following result is well-known.

Theorem 3.1 (Mumford [40]). For every $\varepsilon>0$, the locus $\mathcal{M}_{g}^{\varepsilon}$ of $\mathcal{M}_{g}$ consisting of Riemann surfaces $S$, such that their shortest nontrivial closed geodesic has length $\ell_{\text {sys }}^{\text {hyp }}(S) \geq \varepsilon$ is compact.

Thus, we are given a very natural and geometric exhaustion function.

Corollary 3.1. $\left(\ell_{\mathrm{sys}}^{\mathrm{hyp}}\right)^{-2}: \mathcal{M}_{g} \rightarrow \mathbb{R}$ is an exhaustion function and it locally agrees with the maximum of finitely many real-analytic functions of type $\left(\ell_{\gamma}^{\text {hyp }}\right)^{-2}$.

In order to pursue strategy $\left(4_{\text {Dol }}\right)$, we need to compute the complex Hessian of $\left(\ell_{\mathrm{sys}}^{\mathrm{hyp}}\right)^{-2}$. Certainly, the fact that such a function is not $C^{2}$ might require some smoothing argument, but a big part of the calculation should boil down to compute $i \partial \bar{\partial}\left(\ell_{\gamma}^{\text {hyp }}\right)^{-2}$ for each fixed $\gamma$. This calculation has already been performed, but the upshot is not what we hoped for.

Theorem 3.2 (Wolpert [53]). The complex Hessian of the locally defined function $\left(\ell_{\gamma}^{\text {hyp }}\right)^{-1}$ is strictly negative-definite.

Hence, $i \partial \bar{\partial}\left(\ell_{\gamma}^{\text {hyp }}\right)^{-2}$ has positive index at most 1 and so using the hyperbolic systole to pursue strategy $\left(4_{\mathrm{Dol}}\right)$ is not possible. On the other hand, $\left(4_{\mathrm{dR}}\right)$ turns out to be not so easy to implement either (see [47], for instance): the attempt proved to be successful in the case of $\mathcal{M}_{2,1}$ (see [48]). 


\subsection{Flat surfaces}

Being plurisuperharmonic (i.e. having negative-definite complex Hessian), hyperbolic length functions proved not so useful for $\left(4_{\mathrm{Dol}}\right)$ but, one can hope that flat length functions work better.

By a result by Troyanov [50], we can endow every Riemann surface $(S, p)$ with one marked point with a canonical flat metric $h$, that forms a conical singularity of angle $(2 g-1) 2 \pi$ at $p$. Moreover, such $h$ is unique up to rescaling.

Thus, if $A(h)$ is the area and $\ell_{\text {sys }}(h)$ is the systole of $h$, then the quantity $A(h) \cdot \ell_{\text {sys }}(h)^{-2}$ is invariant under rescaling and work [28] by Kerckhoff-MasurSmillie yields the following.

Corollary 3.2. $\left(A \cdot \ell_{\text {sys }}^{-2}\right): \mathcal{M}_{g, 1} \rightarrow \mathbb{R}$ is an exhaustion function and it locally agrees with the maximum of finitely many real-analytic functions of type $\left(A \cdot \ell_{\gamma}^{-2}\right)$.

Since $i \partial \bar{\partial}\left(e^{F}\right)=i e^{F}[\partial \bar{\partial} F+\partial F \wedge \bar{\partial} F]$, the index of positivity of $i \partial \bar{\partial} F$ is either the same as that of $i \partial \bar{\partial}\left(e^{F}\right)$ or one less. Thus, analyzing the complex Hessian of $A \cdot \ell_{\text {sys }}^{-2}$ is almost the same as treating $i \partial \bar{\partial} \log (A)-i \partial \bar{\partial} \log \left(\ell_{\text {sys }}^{2}\right)$.

A natural way to locally study such functions is the following.

Consider a surface $(S, p) \in \mathcal{M}_{g, 1}$ and fix a universal cover $\pi: \widetilde{S} \rightarrow S$ and a point $\tilde{p} \in \widetilde{P}:=\pi^{-1}(p)$. The canonical flat metric $h$ lifts to a flat metric $\tilde{h}$ on $\widetilde{S}$, with conical singularities at all points in $\widetilde{P}$. Thus, there exists a holomorphic differential $\tilde{\varphi}$ on $\widetilde{S}$ with zeroes of order $2 g-2$ at $\widetilde{P}$, such that $|\tilde{\varphi}|^{2}=\tilde{h}$. Moreover, there exists a holonomy representation $\rho: \pi_{1}(S) \rightarrow \mathrm{U}(1)$, such that an element $\alpha \in \pi_{1}(S)$ acts on $\widetilde{S}$ via a biholomorphism $\tau_{\alpha}: \widetilde{S} \rightarrow \widetilde{S}$ that satisfies $\tau_{\alpha}^{*}(\tilde{\varphi})=\rho(\alpha)^{-1} \tilde{\varphi}$.

Given a one-parameter holomorphic family $\varepsilon \mapsto\left(S_{\varepsilon}, p_{\varepsilon}\right) \in \mathcal{M}_{g, 1}$ of one-pointed Riemann surfaces with $\left(S_{0}, p_{0}\right)=(S, p)$, we can biholomorphically identify all universal covers of $S_{\varepsilon}$ to the same $\widetilde{S}$. Thus, there is a family of flat canonical metrics $h_{\varepsilon}$ that lifts to $\tilde{h}_{\varepsilon}$ on $\widetilde{S}$ and so there are induced one-parameter families of points $\widetilde{P}_{\varepsilon}$ and of holomorphic differentials $\tilde{\varphi}_{\varepsilon}$ on $\widetilde{S}$, such that $\left|\tilde{\varphi}_{\varepsilon}\right|^{2}=\tilde{h}_{\varepsilon}$. Whereas the position of the points $\widetilde{P}_{\varepsilon}$ varies holomorphically with $\varepsilon$, we stress that $\tilde{h}_{\varepsilon}$ need not depend holomorphically on $\varepsilon$, if $\rho_{\varepsilon}$ is not constantly equal to $\rho$.

Let $\gamma$ be a shortest closed curve on $S$, which we may assume that starts and ends at $p$, but does not meet $p$ elsewhere (otherwise it would not be shortest). It can be lifted to a curve $\tilde{\gamma}$ between two distinct points $\tilde{p}, \tilde{p}^{\prime} \in \tilde{P}$. Hence,

$$
\ell_{\gamma}\left(h_{\varepsilon}\right)^{2}=\left|\int_{\tilde{p}_{\varepsilon}}^{\tilde{p}_{\varepsilon}^{\prime}} \tilde{\varphi}_{\varepsilon}\right|^{2}
$$

and so $\varepsilon \mapsto \ell_{\gamma}\left(h_{\varepsilon}\right)^{2}$ is the squared norm of a holomorphic function, if the holonomy representation $\rho_{\varepsilon}$ is independent of $\varepsilon$. In this latter case, $\varepsilon \mapsto \log \left(\ell_{\gamma}^{-2}\left(h_{\varepsilon}\right)\right)$ is harmonic and so $\varepsilon \mapsto \log \left(\ell_{\text {sys }}^{-2}\left(h_{\varepsilon}\right)\right)$ is weakly subharmonic, because the maximum of subharmonic functions is subharmonic. 
In order to obtain more positivity from length functions, we can consider more curves instead of just the shortest one. The problem is that a flat geodesic $\gamma$ for $h$ may not be smooth, that is it may pass through $p$, thus breaking into a union of finitely many segments $\gamma^{(k)}$ : in this case, the length function associated to the path $\gamma$ locally agrees with the sum of the length functions $\ell_{\gamma^{(k)}}$. Thus, even assuming that $\rho_{\varepsilon}$ does not vary and so $\varepsilon \mapsto \ell_{\gamma^{(k)}}\left(h_{\varepsilon}\right)$ is locally the absolute value of a holomorphic function, $\varepsilon \mapsto \ell_{\gamma}^{-2}\left(h_{\varepsilon}\right)$ is weakly subharmonic near $\varepsilon=0$ if $\gamma$ is smooth, but it need not be if $\gamma$ is not smooth.

A way to get around this latter problem is to consider the set $\mathcal{B}$ of $2 g$-ples $B=\left\{\gamma_{1}, \ldots, \gamma_{2 g}\right\}$ of simple closed curves on $S$ based at $p$ that generate $H_{1}(S, p ; \mathbb{R})$ and to define

$$
\ell_{\mathcal{B}}^{-2}=\max _{B \in \mathcal{B}} \ell_{B}^{-2}, \quad \ell_{B}^{-2}=\sum_{\gamma \in B} \ell_{\gamma}^{-2} .
$$

For small $\varepsilon$, the value of $\ell_{\mathcal{B}}^{-2}\left(h_{\varepsilon}\right)$ is attained at finitely many bases $B$ made of smooth $h$-geodesics. Thus, $\varepsilon \mapsto \ell_{\mathcal{B}}^{-2}\left(h_{\varepsilon}\right)$ is again subharmonic, if $\rho_{\varepsilon}$ does not vary.

A harder problem is to study the Laplacian of $\varepsilon \mapsto A\left(h_{\varepsilon}\right)$. Again, the situation is simpler, if the holonomy $\rho_{\varepsilon}$ does not vary: this computation was performed by Veech [51]. The calculation for deformations that truly vary the holonomy is still missing. A simple way to ensure that the holonomy is constant in $\varepsilon$ is to require that $\rho_{\varepsilon}$ takes values at torsion points of $\mathrm{U}(1)$ for every $\varepsilon$.

Remark 3.2. Notice that a flat metric with $r$-torsion linear holonomy is of type $|\psi|^{\frac{2}{r}}=(\psi \bar{\psi})^{\frac{1}{r}}$, where $\psi$ is a nonzero holomorphic section of $K_{S}^{\otimes r}$. In particular, flat surfaces with trivial linear holonomy, namely couples $(S, \varphi)$ with $0 \neq \varphi \in H^{1,0}(S)$, are also called "translation surfaces".

It will be clear in the next section, that the locus of pointed surfaces $(S, p) \in$ $\mathcal{M}_{g, 1}$ such that the canonical flat metric $h$ has trivial holonomy is a closed complex algebraic subvariety of codimension $g-1$.

One can study flat surfaces with more than one singularity in a similar fashion, again using Troyanov's result, which here we state more formally.

Theorem 3.3 (Troyanov [50]). Let $\boldsymbol{m}=\left(m_{1}, \ldots, m_{k}\right)$ be a positive vector, such that $m_{1}+\cdots+m_{k}=2 g-2$. Every $\left(S, p_{1}, \ldots, p_{k}\right) \in \mathcal{M}_{g, k}$ can be endowed with a conformal flat metric $h$ with conical singularity of angle $2 \pi\left(m_{i}+1\right)$ at $p_{i}$ and such a metric is unique up to rescaling. Moreover, the metric $h$ with unit area depends real-analytically on the complex structure of $\left(S, p_{1}, \ldots, p_{k}\right)$.

Analogously, the locus of flat surfaces with torsion or trivial holonomy plays a special role.

Definition 3.2. Let $\boldsymbol{m}=\left(m_{1}, \ldots, m_{k}\right)$ be a positive integral vector such that $m_{1}+\cdots+m_{k}=2 g-2$. The moduli space of translation surfaces of genus $g$ with marked singularities of type $\boldsymbol{m}$ is the space $\Omega \mathcal{M}_{g, k}(\boldsymbol{m})$ that parametrizes 
triples $(S, P, \varphi)$, where $\left(S, P=\left\{p_{1}, \ldots, p_{k}\right\}\right) \in \mathcal{M}_{g, k}$ and $\varphi$ is a nonzero Abelian differential on $S$ that vanishes to order $m_{i}$ at $p_{i}$.

If we consider nonzero Abelian differentials $\varphi$ up to rescaling, we obtain the moduli space of projective translation surfaces $\mathbb{P} \Omega \mathcal{M}_{g, k}(\boldsymbol{m})$. We remark that $\mathbb{P} \Omega \mathcal{M}_{g, k}(\boldsymbol{m})$ agrees with the locus inside $\mathcal{M}_{g, k}$ of pointed surfaces whose associated flat metric $h$ has trivial holonomy. Indeed, for $(S, P, \varphi) \in \Omega_{\mathcal{M}_{g, k}}(\boldsymbol{m})$ the flat metric is recovered as $h=|\varphi|^{2}$ and so its area is simply

$$
A(h)=\frac{i}{2} \int_{S} \varphi \wedge \bar{\varphi} .
$$

It might then be a bit more clear, why the computation of the complex Hessian of $A$ is easier in this case.

\subsection{Translation surfaces with prescribed singularities}

Fix a string $\boldsymbol{m}=\left(m_{1}, \ldots, m_{k}\right)$ of positive integers such that $\sum_{i} m_{i}=2 g-2$. The moduli spaces $\Omega \mathcal{M}_{g, k}(\boldsymbol{m})$ are not connected in general.

Theorem 3.4 (Kontsevich-Zorich [31]). Let $g \geq 2$. The moduli space $\Omega_{\mathcal{M}_{g, k}}$ $(\boldsymbol{m})$ is the disjoint union of $\Omega \mathcal{M}_{g, k}^{\text {hyp }}(\boldsymbol{m})$ and $\Omega \mathcal{M}_{g, k}^{\text {non-hyp }}(\boldsymbol{m})$.

(a) The only nonempty hyperelliptic components are $\Omega \mathcal{M}_{g, 1}^{\text {hyp }}(2 g-2)$ and $\Omega \mathcal{M}_{g, 2}^{\mathrm{hyp}}(g-1, g-1)$ and they consist entirely of hyperelliptic curves. The locus $\Omega \mathcal{M}_{g, k}^{\text {non-hyp }}(\boldsymbol{m})$ is made of components which are generally made of nonhyperelliptic curves. Clearly, there are no nonhyperelliptic components in genus 2.

(b) Let $g \geq 3$ and suppose that the integral vector $\boldsymbol{m}$ is not divisible by 2 . Then $\Omega \mathcal{M}_{g, k}^{\text {non-hyp }}(\boldsymbol{m})$ is nonempty and connected.

(c) Let $\boldsymbol{m}=2 \boldsymbol{n}$, where $\boldsymbol{n}=\left(n_{1}, \ldots, n_{k}\right)$ is integral. For every $(S, P, \varphi) \in$ $\Omega \mathcal{M}_{g, k}^{\text {non-hyp }}(\boldsymbol{m})$, let $L=\mathcal{O}_{S}\left(n_{1} p_{1}+\cdots+n_{k} p_{k}\right)$ be the holomorphic line bundle on $S$ such that $L^{\otimes 2} \cong K_{S}$.

(c1) For $g=3$, the space $\Omega \mathcal{M}_{g, k}^{\text {non-hyp }}(\boldsymbol{m})=\Omega \mathcal{M}_{g, k}^{\text {odd }}(\boldsymbol{m})$ is nonempty and connected.

(c2) For $g \geq 4$, the space $\Omega \mathcal{M}_{g, k}^{\text {non-hyp }}(\boldsymbol{m})$ has two connected components: $\Omega \mathcal{M}_{g, k}^{\text {odd }}(\boldsymbol{m})$ and $\Omega \mathcal{M}_{g, k}^{\text {even }}(\boldsymbol{m})$. The former consists of $(S, P, \varphi)$, such that $h^{0}(S, L)$ is odd; the latter consists of $(S, P, \varphi)$ such that $h^{0}(S, L)$ is even.

Really, if we want to apply strategy $\left(4_{\mathrm{Dol}}\right)$ to estimate the Dolbeault cohomological dimension of $\mathbb{P} \Omega \mathcal{M}_{g, k}(\boldsymbol{m})$, connectedness is not so important (equivalently, we can work with each connected component separately, if we prefer).

Certainly, smoothness of the space is important. The following well-known result was proven in different ways by Hubbard-Masur [25], Veech [51] and Möller [36].

Theorem $3.5([\mathbf{2 5}, \mathbf{5 1}, \mathbf{3 6}])$. The moduli space $\Omega \mathcal{M}_{g, k}(\boldsymbol{m})$ is smooth of dimension $2 g-1+k$. 
The above result can be made more precise by describing special holomorphic charts, known as period coordinates. These will be very useful in the computation of the complex Hessian of $\log \left(A \cdot \ell_{\mathcal{B}}^{-2}\right)$.

Let $U$ be an open subset of $\Omega \mathcal{M}_{g, k}(\boldsymbol{m})$. By the universal property of $\Omega \mathcal{M}_{g, k}(\boldsymbol{m})$, there is an induced holomorphic family $\mathcal{S} \rightarrow U$ of Riemann surfaces of genus $g$ together with disjoint sections $\boldsymbol{p}_{\mathbf{1}}, \ldots, \boldsymbol{p}_{\boldsymbol{k}}: U \rightarrow \mathcal{S}$.

Suppose now that $U$ is contractible. Then the family $\mathcal{S} \rightarrow U$ can be smoothly (but not holomorphically!) trivialized, so that $f: \mathcal{S} \stackrel{\cong}{\longrightarrow} S \times U$ and the section $\boldsymbol{p}_{\boldsymbol{i}}: U \rightarrow \mathcal{S} \cong S \times U$ is constantly equal to a fixed point $p_{i} \in S$. Thus, every point $u$ in $U$ corresponds to a couple $\left(J_{u}, \varphi_{u}\right)$, where $J_{u}$ is a complex structure on $S$ and $\varphi_{u}$ is a $J_{u}$-holomorphic differential one-form on $S$ that vanishes to order $m_{i}$ at $p_{i}$. In particular, $\varphi_{u}$ is closed and so it defines a relative cohomology class $\left(\varphi_{u}\right) \in$ $H^{1}(S, P ; \mathbb{C})$, where $P=\left\{p_{1}, \ldots, p_{k}\right\}$. Hence, there is an induced local period map

$$
\mathcal{P}: U \longrightarrow H^{1}(S, P ; \mathbb{C})
$$

defined as $\mathcal{P}(u):=\left(\varphi_{u}\right)$. Theorem 3.5 can be then made more precise as follows.

Theorem 3.6 ([25, 51, 36]). The map $\mathcal{P}$ is a local biholomorphism. A different choice of the trivialization $f$ corresponds to a post-composition of $\mathcal{P}$ with an integral automorphism of $H^{1}(S, P ; \mathbb{C})$.

By the analysis done in the previous section, the function $\ell_{\mathcal{B}}^{-2}: \Omega \mathcal{M}_{g, k}(\boldsymbol{m}) \rightarrow \mathbb{R}$ is strongly plurisubharmonic, since the maximum is ranging over the set $\mathcal{B}$ of certain $\mathbb{R}$-bases of $H_{1}(S, P ; \mathbb{R})$. Moreover, $i \partial \bar{\partial} \log \left(\ell_{\mathcal{B}}^{-2}\right)$ is strictly positive in any direction different from the ray along which the differential $\varphi$ is rescaled.

On the other hand, the area function $A: \Omega \mathcal{M}_{g, k}(\boldsymbol{m}) \rightarrow \mathbb{R}$ is now easy to study. Indeed, in local period coordinates, it can be expressed as

$$
A(u)=\frac{i}{2} \int_{S} \varphi_{u} \wedge \overline{\varphi_{u}}
$$

and so it is a Hermitian quadratic form on $H^{1}(S, P ; \mathbb{C})$. Hence, by identifying $T_{\left(\varphi_{b}\right)} H^{1}(S, P ; \mathbb{C})$ to $H^{1}(S, P ; \mathbb{C})$, the complex Hessian $i \partial \bar{\partial} A$ can be identified to $A$ itself. Now notice that

$$
\frac{i}{2} \int_{S} \psi \wedge \bar{\psi} \geq 0 \text { for } \psi \text { closed one-form such that } \psi^{0,1} \text { is exact, }
$$

where $\psi^{0,1}$ is the $(0,1)$-component of $\psi$ with respect to the $J_{u}$-holomorphic structure.

We conclude that the Hermitian form $A: H^{1}(S, P ; \mathbb{C}) \rightarrow \mathbb{R}$ has positivity $g$, negativity $g$ and nullity $k-1$, and in particular, we obtain the following.

Lemma 3.2. The function $A$ (respectively $\ell_{\mathcal{B}}^{-2}$ ) satisfies the following weak (respectively strong) (*)-property:

( ) its complex Hessian is non-negative (respectively strictly positive) along deformations $\varepsilon \mapsto\left(J_{\varepsilon}, \varphi+\varepsilon \dot{\varphi}\right)$, such that the $(0,1)$-component (with respect to $J_{0}$ ) of $\dot{\varphi}$ is exact. 
Deformations involved in property $(\star)$ describe a complex subspace of codimension $g$ of the tangent space.

Hence, strategy $\left(4_{\text {Dol }}\right)$ applied to $\mathbb{P} \Omega \mathcal{M}_{g, k}(\boldsymbol{m})$ and the function $\xi_{\boldsymbol{m}}:=A \cdot \ell_{\mathcal{B}}^{-2}$ gives the following estimate.

Corollary 3.3 ([38]). The complex Hessian of the exhaustion function $\xi_{\boldsymbol{m}}$ : $\mathbb{P} \Omega \mathcal{M}_{g, k}(\boldsymbol{m}) \rightarrow \mathbb{R}$ is strictly positive (in the distributional sense) along a smooth distribution of complex codimension $g$ of the tangent bundle. Hence, coh-dim Dol $\left(\mathbb{P} \Omega \mathcal{M}_{g, k}(\boldsymbol{m})\right) \leq g$.

A more careful computation shows that there exist points $(S, P,[\varphi]) \in$ $\mathbb{P} \Omega \mathcal{M}_{g, k}(\boldsymbol{m})$ at which the complex Hessian of $\xi_{\boldsymbol{m}}$ has indeed index of negativity exactly $g$, and so this function can provide no better bound for the cohomological dimension. On the other hand, we know that such an estimate is not optimal.

Proposition 3.1. The following components of the moduli spaces of projective translation surfaces are affine:

(a) $\mathbb{P} \Omega \mathcal{M}_{g, 1}^{\text {hyp }}(2 g-2)$ and $\mathbb{P} \Omega \mathcal{M}_{g, 2}^{\text {hyp }}(g-1, g-1)$;

(b) $\mathbb{P} \Omega \mathcal{M}_{3,1}^{\text {odd }}(4)$ and $\mathbb{P} \Omega \mathcal{M}_{3,2}(3,1)$;

(c) $\mathbb{P} \Omega \mathcal{M}_{4,1}^{\text {odd }}(6), \mathbb{P} \Omega \mathcal{M}_{4,1}^{\text {even }}(6)$ and $\mathbb{P} \Omega \mathcal{M}_{5,1}^{\text {even }}(8)$.

Hence, both their algebraic and Dolbeault cohomological dimensions are zero.

As hyperelliptic curves are branched, double covers of $\mathbb{C P}^{1}$ part (a) can be considered classical. Almost as classical is part (b), which relies on the esplicit description of nonhyperelliptic smooth Riemann surfaces of genus 3 as plane quartics (see, for instance, $[32,34]$ ). During conversations on this topic with Eduard Looijenga, he pointed out that works of Pinkham [42] and Vitulli [52] on deformations of affine monomial curves imply that certain components of type $\mathbb{P} \Omega \mathcal{M}_{g, 1}^{\text {odd }}(2 g-2), \mathbb{P} \Omega \mathcal{M}_{g, 1}^{\text {even }}(2 g-2)$ are the complement of a discriminant hypersurface inside a complete intersection in a weighted projective space, which implies part (c) of the above proposition.

Question 3.1. Are the spaces $\mathbb{P} \Omega \mathcal{M}_{g}(\boldsymbol{m})$ affine?

We will see below, that a positive answer would have consequences on the cohomological dimension of $\mathcal{M}_{g}$.

\subsection{Hodge bundle}

Translation surfaces of genus $g$ without marked points and with no fixed pattern of zeroes are determined by couples $(S, \varphi)$, where $S \in \mathcal{M}_{g}$ and $0 \neq \varphi \in H^{1,0}(S)$. Hence, they are naturally parametrized by the space $\Omega \mathcal{M}_{g}$, an algebraic $\left(\mathbb{C}^{g} \backslash\{0\}\right)$ bundle over $\mathcal{M}_{g}$, whose fiber over $S$ is exactly $H^{1,0}(S) \backslash\{0\}$. Thus, projective translation surfaces without marked points and with no fixed pattern of zeroes are 
parametrized by the projectivization $\mathbb{P} \Omega \mathcal{M}_{g}$, which is an algebraic $\mathbb{C P}^{g-1}$-bundle over $\mathcal{M}_{g}$, and so coh-dim $\operatorname{dim}_{\mathfrak{F}}\left(\mathcal{M}_{g}\right)=\operatorname{coh}-\operatorname{dim}_{\mathfrak{F}}\left(\mathbb{P} \Omega \mathcal{M}_{g}\right)-(g-1)$ for $\mathfrak{F}=$ Dol, alg.

For every $\boldsymbol{m}=\left(m_{1}, \ldots, m_{k}\right)$ string of positive integers with $m_{1}+\cdots+m_{k}=$ $2 g-2$, the map $\mathbb{P} \Omega \mathcal{M}_{g, k}(\boldsymbol{m}) \rightarrow \mathbb{P} \Omega \mathcal{M}_{g}$, that forgets the marked points is finite over its image, which we denote by $\mathbb{P} \Omega \mathcal{M}_{g}(\boldsymbol{m})$.

We want to estimate the Dolbeault cohomological dimension of $\mathbb{P} \Omega \mathcal{M}_{g}$, which is stratified by subvarieties $\mathbb{P} \Omega \mathcal{M}_{g}(\boldsymbol{m})$ of codimension $2 g-2-k$.

Remark 3.3. The stratum $\mathbb{P} \Omega \mathcal{M}_{g}(\boldsymbol{m})$ is affine if and only if $\mathbb{P} \Omega \mathcal{M}_{g, k}(\boldsymbol{m})$ is. If Question 3.1 has a positive answer, then $\left(2_{\text {alg }}\right)$ implies that coh-dimalg $\left(\mathbb{P} \Omega \mathcal{M}_{g}\right) \leq$ $2 g-3$ and so coh-dimalg $\left(\mathcal{M}_{g}\right) \leq(2 g-3)-(g-1)=g-2$.

Unfortunately, dealing with Dolbeault cohomology, we cannot invoke the line of proof $\left(2_{\mathrm{alg}}\right)$, but we need to use strategy $\left(1_{\text {Dol }}\right)$. Having already an inspiring stratification of $\mathbb{P} \Omega \mathcal{M}_{g}$, the rough idea is to proceed as follows:

(i) thicken each stratum $\mathbb{P} \Omega \mathcal{M}_{g}(\boldsymbol{m})$ to an open subset $U_{\boldsymbol{m}}$ in such a way that $U_{\boldsymbol{m}}$ and $U_{\boldsymbol{m}^{\prime}}$ intersect, if and only if, one stratum is contained in the closure of the other;

(ii) for every $\boldsymbol{m}$, extend $\xi_{\boldsymbol{m}}$ to $U_{\boldsymbol{m}}$, so that $i \partial \bar{\partial} \xi_{\boldsymbol{m}}$ is non-negative along a $(2 g-$ $2-k)$-codimensional distribution that complements $T \mathbb{P} \Omega \mathcal{M}_{g}(\boldsymbol{m})$;

(iii) define $\zeta_{\boldsymbol{m}}: U_{\boldsymbol{m}} \rightarrow \mathbb{R}$ with the following properties:

(a) $\zeta_{\boldsymbol{m}}$ is non-negative and it vanishes on $\mathbb{P} \Omega \mathcal{M}_{g}(\boldsymbol{m})$,

(b) there exists a constant $c>0$ independent of $\boldsymbol{m}$ such that $\liminf (C, P,[\varphi])$ $\zeta_{\boldsymbol{m}} \geq c>0$ for every $(C, P,[\varphi]) \in \bar{U}_{\boldsymbol{m}} \backslash \overline{\mathbb{P} \Omega \mathcal{M}_{g}(\boldsymbol{m})}$,

(c) the complex Hessian of $\zeta_{\boldsymbol{m}}$ is strictly positive in the directions transverse to $\mathbb{P} \Omega \mathcal{M}_{g}(\boldsymbol{m})$,

(iv) define a smaller open neighborhood $V_{\boldsymbol{m}}=\left\{\zeta_{\boldsymbol{m}}<c^{\prime}\right\} \subset U_{\boldsymbol{m}}$ of $\mathbb{P} \Omega \mathcal{M}_{g}(\boldsymbol{m})$ for a suitable $c^{\prime} \in(0, c)$;

(v) consider the function $\hat{\xi}_{m}:=\xi_{m}+\chi \circ \zeta_{m}$, where $\chi>0$ satisfies $\chi(0)=1$, $\chi^{\prime}>0$ and $\chi^{\prime \prime}>0$.

Suppose that the above steps are accomplished. It follows that $\hat{\xi}_{\boldsymbol{m}}: V_{\boldsymbol{m}} \rightarrow \mathbb{R}$ is an exhaustion function and a quick computation shows that the $i \partial \bar{\partial} \hat{\xi}_{m}$ satisfies a suitable phrasing of the strong $(\star)$ property.

Consider now the open cover $\mathfrak{V}=\left\{V_{\boldsymbol{m}}\right\}$ of $\mathbb{P} \Omega \mathcal{M}_{g}$. Every intersection of open sets in $\mathfrak{V}$ is of type $V_{\boldsymbol{m}^{0}} \cap \cdots \cap V_{\boldsymbol{m}^{l}}$, where $\mathbb{P} \Omega \mathcal{M}_{g}\left(\boldsymbol{m}^{h}\right)$ is in the closure of $\mathbb{P} \Omega \mathcal{M}_{g}\left(\boldsymbol{m}^{\boldsymbol{h}-\mathbf{1}}\right)$ for $h=1, \ldots, l$. Observe finally that:

(a) $\hat{\xi}_{\boldsymbol{m}^{0}}+\cdots+\hat{\xi}_{\boldsymbol{m}^{l}}$ is an exhaustion function on $V_{\boldsymbol{m}^{\mathrm{o}}} \cap \cdots \cap V_{\boldsymbol{m}^{l}}$ that satisfies strong $(\star)$ and so coh-dim $\operatorname{dol}\left(V_{\boldsymbol{m}^{\mathrm{o}}} \cap \cdots \cap V_{\boldsymbol{m}^{l}}\right) \leq g$;

(b) a nonempty intersection of open sets in $\mathfrak{V}$ involves at most $2 g-2$ elements.

Hence, $\left(1_{\text {Dol }}\right)$ allows to conclude as follows.

Theorem 3.7 ([38]). coh-dim $\operatorname{Dol}\left(\mathbb{P} \Omega \mathcal{M}_{g}\right) \leq 3 g-3$. 
Recalling that $\mathbb{P} \Omega \mathcal{M}_{g} \rightarrow \mathcal{M}_{g}$ is a holomorphic $\mathbb{C} \mathbb{P}^{g-1}$-fibration, we have the following estimate.

Corollary 3.4 ([38]). coh- $\operatorname{dim}_{\text {Dol }}\left(\mathcal{M}_{g}\right) \leq 2 g-2$.

We underline that, analogously to the case of those affine strata (see Proposition 3.1) for which Corollary 3.3 provided a nonoptimal upper bound to the Dolbeault cohomological dimension, for $\mathcal{M}_{g}$ too the above result remains $g$ steps off a positive solution to Looijenga's problem.

\section{Acknowledgments}

The author thanks the organizers Dirk Kreimer, Holger Reich and Alexander Schmitt of VBAC 2014 Workshop at the Freie Universität Berlin for the invitation and for the warm and fruitful atmosphere. The author's research was partially supported by Grant FIRB 2010 (RBFR10GHHH_003) "Low-dimensional geometry and topology".

\section{References}

[1] W. Abikoff, The Real Analytic Theory of Teichmüller Space, Lecture Notes in Mathematics, Vol. 820 (Springer, Berlin, 1980).

[2] Y. Akizuki and S. Nakano, Note on Kodaira-Spencer's proof of Lefschetz theorems, Proc. Japan Acad. 30 (1954) 266-272.

[3] A. Andreotti and H. Grauert, Théorème de finitude pour la cohomologie des espaces complexes, Bull. Soc. Math. France 90 (1962) 193-259.

[4] E. Arbarello and M. Cornalba, Calculating cohomology groups of moduli spaces of curves via algebraic geometry, Publ. Math. Inst. Hautes Études Sci. 88(1) (1998) 97-127.

[5] E. Arbarello, M. Cornalba and P. A. Griffiths, Geometry of Algebraic Curves, Grundlehren der Mathematischen Wissenschaften [Fundamental Principles of Mathematical Sciences], Vol. 268 (Springer, Heidelberg, 2011).

[6] L. Bers, A non-standard integral equation with applications to quasiconformal mappings, Acta Math. 116 (1966) 113-134.

[7] R. Bieri and B. Eckmann, Groups with homological duality generalizing Poincaré duality, Invent. Math. 20 (1973) 103-124.

[8] S. Bochner, Curvature and Betti numbers, Ann. Math. (2) 49 (1948) 379-390.

[9] S. Bochner, Curvature and Betti numbers. II, Ann. Math. (2) 50 (1949) 77-93.

[10] S. Boldsen, Improved homological stability for the mapping class group with integral or twisted coefficients, Math. Z. 270(1-2) (2012) 297-329.

[11] P. Deligne and D. Mumford, The irreducibility of the space of curves of given genus, Publ. Math. Inst. Hautes Études Sci. 36 (1969) 75-109.

[12] J.-P. Demailly, Complex Analytic and Differential Geometry, OpenContent Book, http://www-fourier.ujf-grenoble.fr/ demailly/manuscripts/agbook.pdf.

[13] S. Diaz, A bound on the dimensions of complete subvarieties of $\mathcal{M}_{g}$, Duke Math. J. 51(2) (1984) 405-408.

[14] D. Edidin, The codimension-two homology of the moduli space of stable curves is algebraic, Duke Math. J. 67(2) (1992) 241-272.

[15] C. Fontanari and S. Pascolutti, An affine open covering of $\mathcal{M}_{g}$ for $g \leq 5$, Geom. Dedicata 158 (2012) 61-68. 
[16] J. Girbau, Sur les théorèmes d'annulation de Kodaira, C. R. Acad. Sci. Paris Sér. $A-B 272$ (1971) A740-A742.

[17] J. Girbau, Sur les théorèmes d'annulation de Kodaira, C. R. Acad. Sci. Paris Sér. $A-B 273$ (1971) A461-A462.

[18] T. Graber and R. Vakil, Relative virtual localization and vanishing of tautological classes on moduli spaces of curves, Duke Math. J. 130(1) (2005) 1-37.

[19] A. Grothendieck, Techniques de Construction en Géométrie Analytique X: Construction de l'espace de Teichmüller, Séminaire Henri Cartan, Vol. 13 (Secr'etariat Mathématique, Paris, 1960-1961), pp. 1-20.

[20] J. Harer, The second homology group of the mapping class group of an orientable surface, Invent. Math. 72(2) (1983) 221-239.

[21] J. Harer, Stability of the homology of the mapping class groups of orientable surfaces, Ann. Math. (2) 121(2) (1985) 215-249.

[22] J. Harer, The virtual cohomological dimension of the mapping class group of an orientable surface, Invent. Math. 84(1) (1986) 157-176.

[23] J. Harer, The third homology group of the moduli space of curves, Duke Math. J. 63(1) (1991) 25-55.

[24] J. Harer and D. Zagier, The Euler characteristic of the moduli space of curves, Invent. Math. 85(3) (1986) 457-485.

[25] J. Hubbard and H. Masur, Quadratic differentials and foliations, Acta Math. 142(3-4) (1979) 221-274.

[26] E.-N. Ionel, Topological recursive relations in $H^{2 g}\left(\mathcal{M}_{g, n}\right)$, Invent. Math. 148(3) (2002) 627-658.

[27] N. Ivanov, On the Homology Stability for Teichmüller Modular Groups: Closed Surfaces and Twisted Coefficients, Mapping class groups and moduli spaces of Riemann surfaces (Göttingen, 1991/Seattle, 1991), Contemporary Mathematics, Vol. 150 (American Mathematical Society, Providence, 1993), pp. 149-194.

[28] S. Kerckhoff, H. Masur and J. Smillie, Ergodicity of billiard flows and quadratic differentials, Ann. Math. (2) 124(2) (1986) 293-311.

[29] K. Kodaira, On Kähler varieties of restricted type (an intrinsic characterization of algebraic varieties), Ann. Math. (2) 60 (1954) 28-48.

[30] M. Kontsevich, Intersection theory on the moduli space of curves and the matrix Airy function, Comm. Math. Phys. 147(1) (1992) 1-23.

[31] M. Kontsevich and A. Zorich, Connected components of the moduli spaces of Abelian differentials with prescribed singularities, Invent. Math. 153(3) (2003) 631-678.

[32] E. Looijenga, Cohomology of $\mathcal{M}_{3}$ and $\mathcal{M}_{3,1}$, Mapping Class Groups and Moduli Spaces of Riemann Surfaces (Göttingen, 1991/Seattle, 1991), Contemporary Mathematics, Vol. 150 (American Mathematical Society, Providence, 1993), pp. 205-228.

[33] E. Looijenga, On the tautological ring of $\mathcal{M}_{g}$, Invent. Math. 121(2) (1995) 411-419.

[34] E. Looijenga and G. Mondello, The fine structure of the moduli space of abelian differentials in genus 3, Geom. Dedicata 169 (2014) 109-128.

[35] I. Madsen and M. Weiss, The stable moduli space of Riemann surfaces: Mumford's conjecture, Ann. Math. (2) 165(3) (2007) 843-941.

[36] M. Möller, Linear manifolds in the moduli space of one-forms, Duke Math. J. 144(3) (2008) 447-487.

[37] G. Mondello, A remark on the homotopical dimension of some moduli spaces of stable Riemann surfaces, J. Eur. Math. Soc. (JEMS) 10(1) (2008) 231-241.

[38] G. Mondello, On the cohomological dimension of the moduli space of Riemann surfaces, to appear on Duke Math J. 
[39] G. Mondello, Stratifications of the moduli space of curves and related questions, Rend. Mat. Appl. (7) 36 (2014) 1-26.

[40] D. Mumford, A remark on Mahler's compactness theorem, Proc. Amer. Math. Soc. 28 (1971) 289-294.

[41] R. C. Penner, Perturbative series and the moduli space of Riemann surfaces, J. Differential Geom. 27(1) (1988) 35-53.

[42] H. Pinkham, Deformations of Algebraic Varieties with $\mathbb{G}_{m}$-Action, Vol. 20 (Société Mathématique de France, Paris, 1974).

[43] H. Poincaré, Les fonctions fuchsiennes et l'équation $\Delta u=e^{u}$, J. Math. Pures Appl. (5e série) 4 (1898) 137-230.

[44] M. Polito, The fourth tautological group of $\overline{\mathcal{M}}_{g, n}$ and relations with the cohomology, Atti Accad. Naz. Lincei Cl. Sci. Fis. Mat. Natur. Rend. Lincei Mat. Appl. (9) 14(2) (2003) 137-168.

[45] J. Powell, Homeomorphisms of $S^{3}$ leaving a Heegaard surface invariant, Trans. Amer. Math. Soc. 257(1) (1980) 193-216.

[46] M. Roth and R. Vakil, The affine stratification number and the moduli space of curves, Algebraic Structures and Moduli Spaces, CRM Proceedings \& Lecture Notes, Vol. 38 (American Mathematical Society, Providence, 2004), pp. 213-227.

[47] P. S. Schaller, Systoles and topological Morse functions for Riemann surfaces, J. Differential Geom. 52(3) (1999) 407-452.

[48] P. S. Schaller, A systolic geometric cell decomposition for the space of once-holed Riemann surfaces of genus 2, Topology 40(5) (2001) 1017-1049.

[49] O. Tommasi, Rational cohomology of the moduli space of genus 4 curves, Compos. Math. 141(2) (2005) 359-384.

[50] M. Troyanov, Les surfaces euclidiennes à singularités coniques, Enseign. Math. (2) 32(1-2) (1986) 79-94.

[51] W. Veech, Flat surfaces, Amer. J. Math. 115(3) (1993) 589-689.

[52] M. Vitulli, The obstruction of the formal moduli space in the negatively graded case, Pacific J. Math. 82(1) (1979) 281-294.

[53] S. Wolpert, Convexity of geodesic-length functions: A reprise, Spaces of Kleinian Groups, London Mathematical Society Lecture Note Series, Vol. 329 (Cambridge University Press, Cambridge, 2006), pp. 233-245. 